\title{
Evaluation of Welding Residual Stresses Using Diffraction Spot Trace Method
}

\author{
Kenji Suzuki $^{1, \mathrm{a}}$, Takahisa Shobu ${ }^{2, \mathrm{~b}}$, Ayumi Shiro $^{2, \mathrm{c}}$ and Shuoyuan Zhang ${ }^{2, \mathrm{~d}}$ \\ ${ }^{1}$ Factulty of Education, Niigata University, \\ 8050, Igarashi-2-no-cho, Nishi-ku, Niigata, 950-2181, Japan \\ ${ }^{2}$ Quantum Beam Science Directorate, Japan Atomic Energy Agency, \\ 1-1-1, Koto, Sayo, Hyogo, 679-5148, Japan \\ asuzuki@ed.niigata-u.ac.jp, bshobu@sp8sun.spring8.or.jp, cshiro.ayumi@jaea.go.jp, \\ dzhang.shuoyuan@jaea.go.jp
}

Keywords: welding residual stress, austenitic stainless steel, synchrotron X-ray, DSTM, rotating slit-system.

\begin{abstract}
A diffraction spot trace method (DSTM) is very useful to measure internal stresses of materials with coarse grains. In this study, validity of the DSTM was examined using high energy synchrotron X-rays of $70 \mathrm{keV}$. The specimen was a welded plate of austenitic stainless steel (SUS316L). The thickness of the specimen was $9 \mathrm{~mm}$. The improved rotating slit-system was used, and PILATUS was used as an area detector. It was difficult to detect the high energy X-rays due to the reduction of counting efficiency of the PILATUS. However, the residual stress map of the welding specimen was obtained by long exposure time. The residual stresses of the welding specimen were simulated by a finite element method (FEM). The outline of the residual stress measured by the DSTM coincided with that by the FEM.
\end{abstract}

\section{Introduction}

X-ray stress measurements were mainly applied to a surface of a welding component of austenitic stainless steel $[1,2]$. Coarse grains, texture and welded parts are objects posing difficulty in an Xray stress measurement, because the diffraction patterns are not rings but spots. Although austenitic stainless steel is important structural material, the measurement of the internal stress is not established because of coarse grains [3]. The combination of the rotating slit-system and the diffraction spot trace method (DSTM) were developed in order to measure internal stress in material with coarse grains [4]. When the X-ray energy exceeds $30 \mathrm{keV}$, the X-ray detection efficiency of PILATUS, which is an area detector, decreases sharply. Therefore, we have applied the DSTM to light alloys, such as aluminum and magnesium alloys using synchrotron radiation with $30 \mathrm{keV}$. The distribution of the internal residual stress in the welded plate of magnesium alloy was obtained using the DSTM, and the validity of the DSTM was checked [5].

The DSTM has not been applied to a stress measurement for the austenitic stainless steel. In this study, internal residual stresses in a welded plate of austenitic stainless steel were measured using the DSTM with hard synchrotron X-rays. It is significant to apply DSTM to hard synchrotron X-rays, though there is apprehension about the performance decrement of PILATUS.

\section{Experimental Method}

Welding Specimen. Material of the specimen is austenitic stainless steel, SUS316L. The shape of the specimen is a square plate of $100 \mathrm{~mm}$ with a thickness of $9 \mathrm{~mm}$ as shown in Fig. 1 (a). We used not a butted plate but an U-notch plate in order to control deformation by welding. The U-notch was machined on the centre of the plate specimen and the detail of the U-notch is shown in Fig. 1 (b).

TIG (tungsten inert gas) welding of the U-notch was carried out. The material of the weld wire was the same SUS316L as the plate. The notch was filled up with three passes of TIG welding. For 


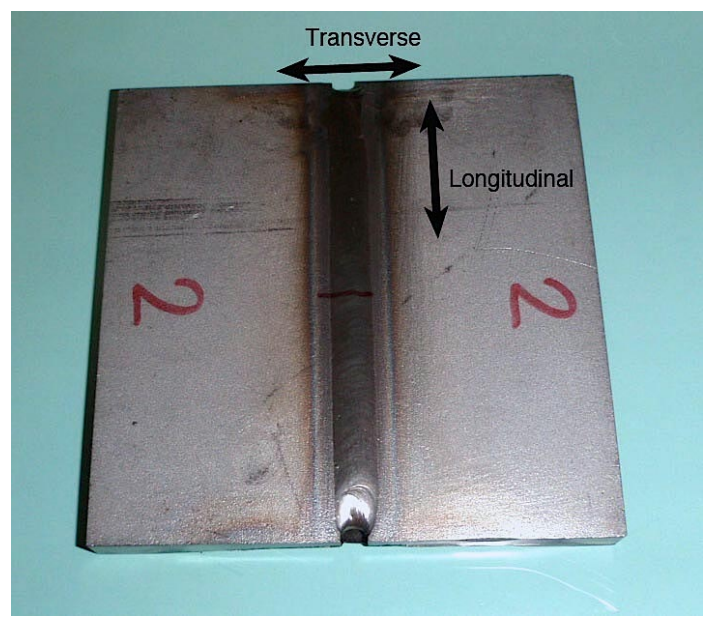

(a) Welding specimen
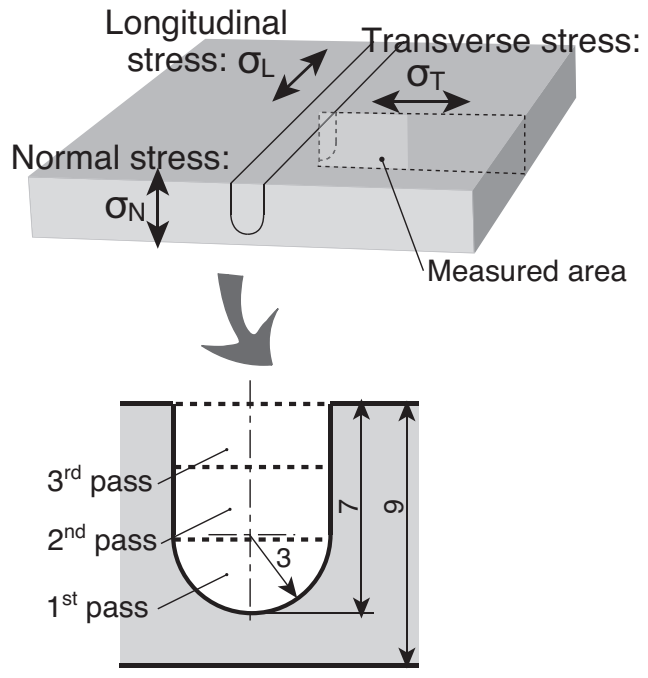

(b) U-notch and stress directions

Fig. 1: U-notch and coordinate system for welding specimen

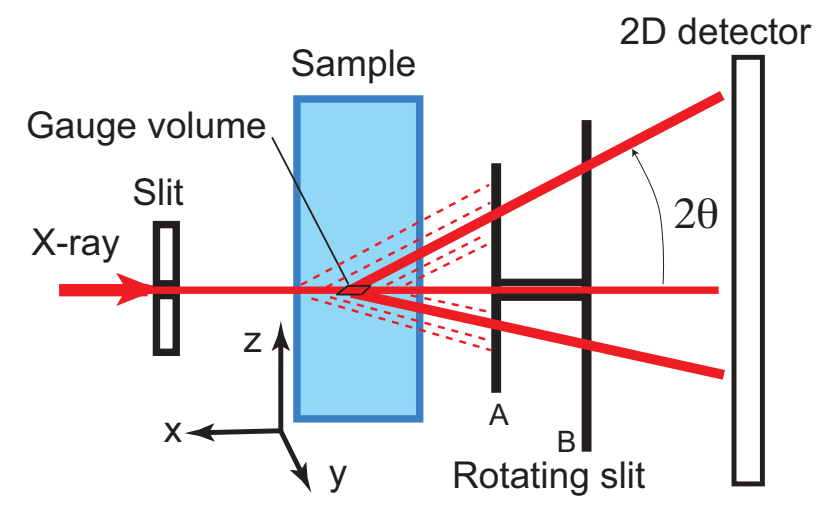

Fig. 2: Rotating slit-system.

the $1 \mathrm{st}$ pass, the voltage and the current were $10 \mathrm{~V}$ and $120 \mathrm{~A}$. The torch speed was $1.5 \mathrm{~mm} / \mathrm{s}$, and the wire feed rate was $0.103 \mathrm{~g} / \mathrm{s}$. For the 2 nd and $3 \mathrm{rd}$ passes, the voltage and the current were $10 \mathrm{~V}$ and $150 \mathrm{~A}$. The torch speed was $1.5 \mathrm{~mm} / \mathrm{s}$, and the wire feed rate was $0.147 \mathrm{~g} / \mathrm{s}$.

Stress Measurement. The residual stresses were measured using hard synchrotron X-rays. The used beam line was BL22XU in SPring-8. X-ray energy was $70.031 \mathrm{keV}$. The diffraction spots were measured by the PILATUS-100K, which was a 2-dimensional proportional counter. The pixcel sizes of PILATUS are $487 \times 195 \mathrm{~mm}^{2}$ and the spatial resolution was $0.172 \mathrm{~mm} /$ pixel.

As shown in Fig. 2, the positions of the detector have to focus on the centre of the gauge volume. Only the diffractions from the gauge volume can reach the detector through the rotating slits A and B. Archimedes' spiral was used as the slit curve. The slit, A and B are similar. A whole diffraction image can be measured by rotating the slit, A and B in the same axis. The rotation rate of the improved rotating slit-system was $2 \mathrm{~Hz}$. This slit-system is called the rotating slit-system in this paper. The details of the rotating slit-system are described in a previous study [4]. The width of each slit is 0.2 $\mathrm{mm}$ and the length of the gauge volume is $2.4 \mathrm{~mm}$ for the diffraction angle $2 \theta=9.36^{\circ}$ of $\gamma$-Fe 311 .

Figure 3 explains the mechanism of the DSTM. The incident X-ray passes through the specimen and the diffracted X-rays from the gauge volume reach the area detector through the rotating slitsystem. The welding specimen on the scanning stage moves from depth A to C with step motion. The diffraction angle and the intensity are measured for each step. The changes in the intensity and the diffraction angle are arranged against the scanning depth, for example, as shown in the right-side 

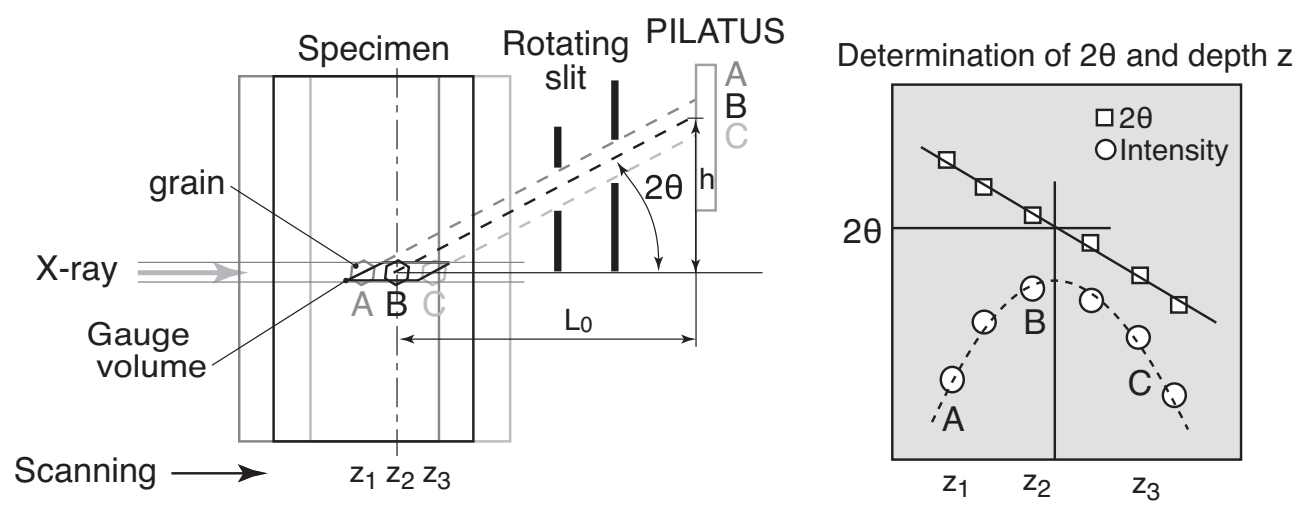

Fig. 3: Experimental system for DSTM.

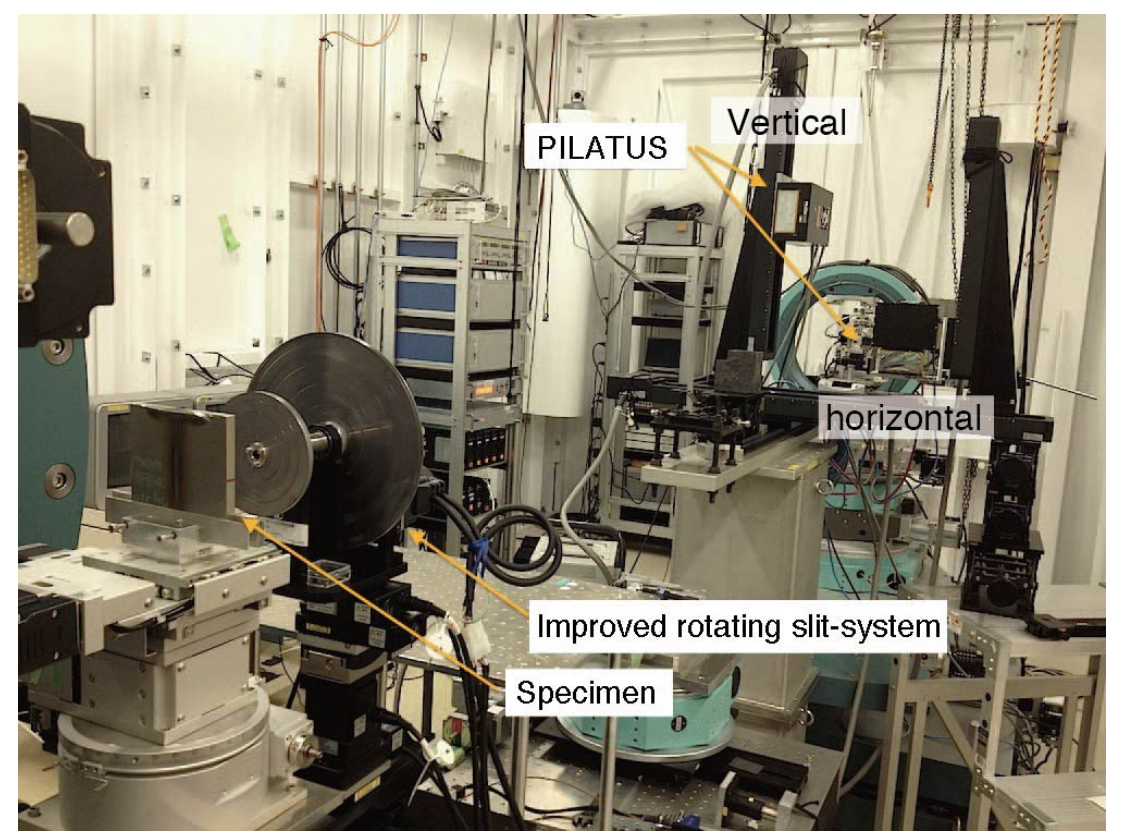

Fig. 4: Experiment for stress measurement using rotating slit-system.

graph in Fig. 3. The peak top of the intensity is obtained by the approximation curve of a parabola. The true diffraction angle $2 \theta$ can be determined at the depth of the peak top of the intensity. In this study, the above method of deterinating the diffraction angle for coarse grains is called the DSTM (diffraction spot trace method).

\section{Results and Discussion}

Residual Stresses in Welding Specimen. Figure 4 shows the experiment for the stress measurement using the rotating slit-system. The weld specimen is mounted on a 3-axis scanning stage. The distance beween the diffraction centre and the detector, $L_{0}$, is approximately $1980 \mathrm{~mm}$. The PILATUS are set in the horizontal and vertical directions, and each detector is mounted on the $x-y$ stage so that a wide area is detected, as shown in the figure.

The long detection time was needed to get the diffraction image with the PILATUS due to the high energy of $70.031 \mathrm{keV}$. So the exposure time of the PILATUS was $30 \mathrm{~min}$. Examples of the images measured by the PILATUS are shown in Fig. 5 (a) and (b). The longitudinal strain to the welding line, $\varepsilon_{L}$, was calculated from the diffraction angle detected by the vertical detector, and the transverse strain to the welding line, $\varepsilon_{T}$, was calculated from the diffraction angle detected by the horizontal one. 


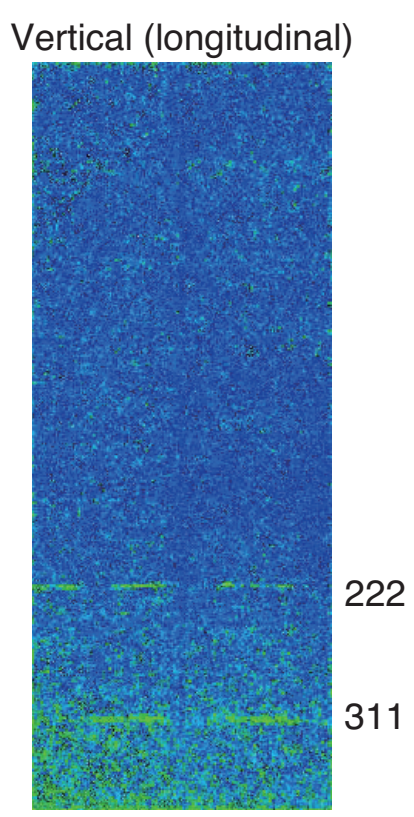

(a) Longitudinal direction

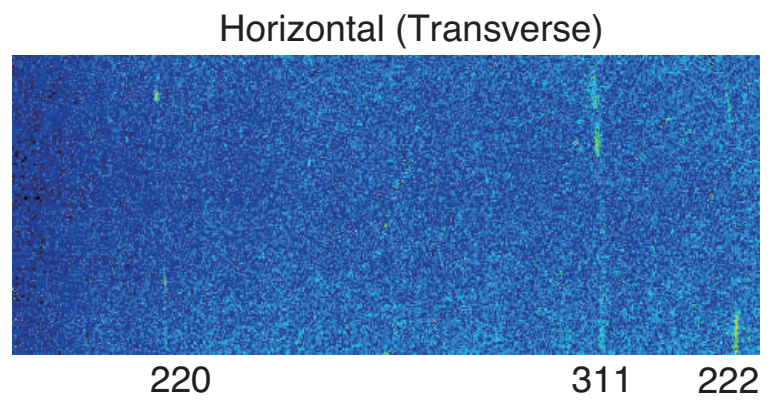

(b) Transverse direction

Fig. 5: PILATUS images of welding specimen. The images (a) and (b) demonstrate the diffractions detected by the vertical and horizontal PILATUS (see Fig. 4).

However, it was difficult to obtain enough intensity to acquire accuracy as shown in Fig. 5 . The peak counts were around 200 counts for exposure $30 \mathrm{~min}$. I tried to use a CCD image sensor other than the PILATUS, but was not able to obtain a satisfactory result. Development of a new CdTe detector for high energy X-rays is desired.

To obtain the residual stress map in the midsection of the welding specimen, the DSTM was carried out. The pitch of the scanning position was $4 \mathrm{~mm}$. The scanning positions were $0,4,8$ and $12 \mathrm{~mm}$ from the welding centre. The scanning stage was moved with a step width of $0.32 \mathrm{~mm} / \mathrm{step}$ from the welding surface to the back surface. Ideally, we wanted to make the step width and the pitch of the scanning position smaller, but were not able to do so. The reason is there was limitation of the beam time.

The diffraction images were measured by the PILATUS, the diffraction angles of the diffraction spots were determined using the DSTM. As the strain free lattice constant $a_{0}$, the mean value of the lattice constant at the position which was far from a weld zone was used. The $a_{0}$-values were $3.59624 \AA$ and $3.59946 \AA$ for the transverse and longitudinal directions, respectively. The 311 and 222 diffractions from $\gamma$-Fe were used. The distribution of the lattice constant $a_{L}$ and $a_{T}$ were approximated with a quartic function. The strains were calculated from $\varepsilon=\left(a-a_{0}\right) / a_{0}$ in $1 \mathrm{~mm}$ square coordinates. The normal strain cannot be measured by synchrotron X-rays because the path length is too large to detect a diffraction. Therefore, the stress was calculated assuming a plane stress state. The used diffraction elastic constants were calculated by Kröner model using stiffnesses of single crystal [6]. The used diffraction elastic constants were $E_{311}=182 \mathrm{GPa}, \nu_{311}=0.307$ for the 311 diffraction and $E_{222}=237 \mathrm{GPa}, \nu_{222}=0.249$ for the 222 diffraction.

The residual stress map of the longitudinal and transverse directions are shown in Fig. 6. For the longitudinal stress $\sigma_{L}$, large tensile stress exists in the weld part and near the notch root. For the transverse stress $\sigma_{T}$, large tensile stress exists near the notch root, and a tension appears near the back surface. Although clear stress maps cannot be obtained since there were few points of measurements, it was significant to obtain the stress map in the welding specimen of austenitic stainless steel.

Simulation of Welding Residual Stresses. In order to evaluate the residual stress measured by the DSTM, a simulation by finite element method (FEM) was carried out. A finite element model that im- 


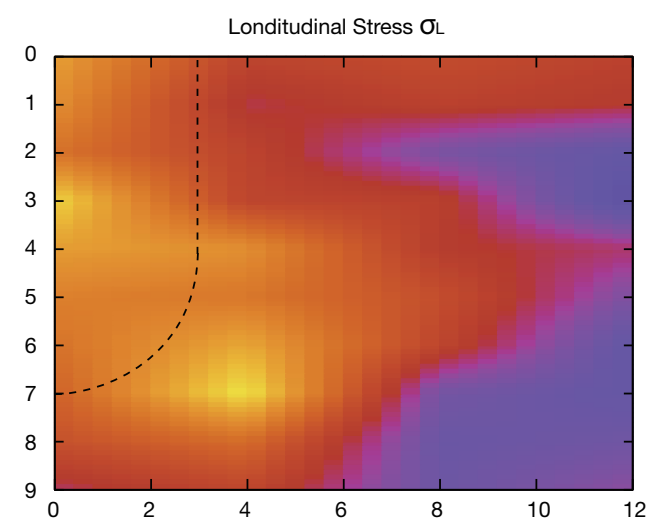

(a) Longitudinal stress $\sigma_{L}$

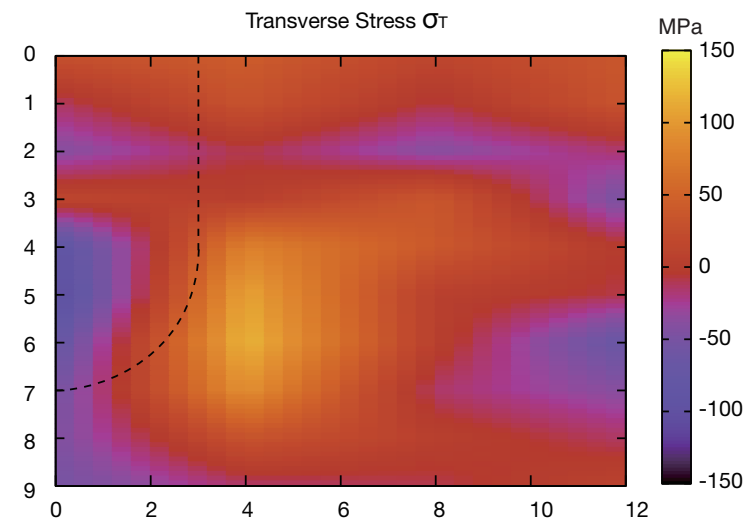

(b) Transverse stress $\sigma_{T}$

Fig. 6: Residual stress maps of welding specimen measured using DSTM. $\sigma_{L}$ and $\sigma_{T}$ are defined in Fig.1 (b). The shape of U-notch is drawn with a broken line.

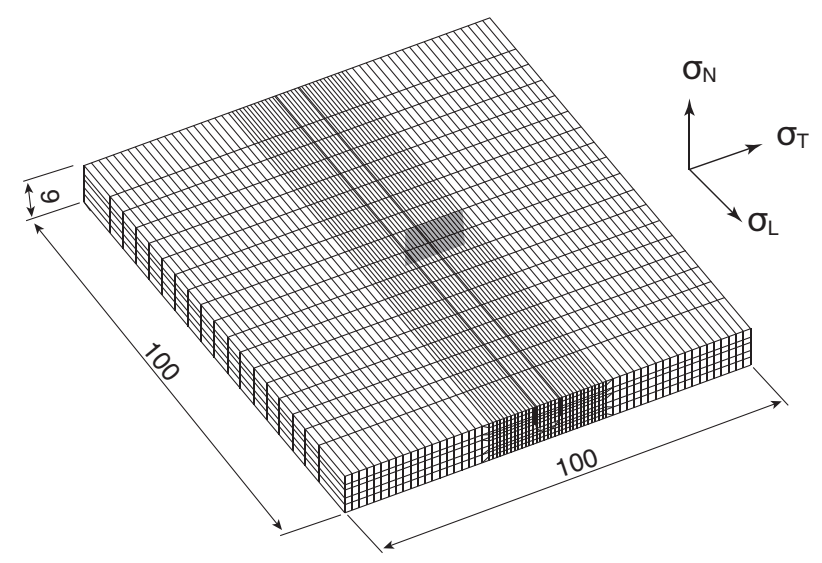

Fig. 7: Finite element model for welding specimen.

itated the welding specimen was made. The finite element model is shown in Fig. 7. The conditions of the simulation were accorded with the welding conditions of the specimen. The temperature and deformation in the welding process was simulated by "Quick Welder" as the FEM tool. The physical properties in the simulation depend on temperature, such as Young's modulus, yield stress, strain-hardening rate, specific heat capacity, thermal conductivity and coefficient of thermal expansion. These values were quoted in another study [7].

The residual stress maps for the welding specimen are shown in Fig. 8. In the figure, (a), (b) and (c) indicate the longitudinal stress, the transverse stress and the normal stress. With respect to the longitudinal residual stress $\sigma_{L}$, large tension arises in the welding zone as shown in Fig. 8 (a). With respect to the transverse stress $\sigma_{T}$, tension arises in the 2nd welding pass. In addition, tensile stress generates near the middle of the U-notch and near the position of about $10 \mathrm{~mm}$ from the centre line of the back surface as shown in the figure (b). For the normal stress $\sigma_{N}$, a small compressive residual stress arises near the notch root as shown in the figure (c). The plane stress state is an appropriate assumption, since the normal residual stress $\sigma_{N}$ is small.

The measured longitudinal stress in Fig. 6 (a) is smaller than the simulation in Fig.8 (a). This difference may be caused by the $a_{0}$-value. Examination is required regarding the determination method of the $a_{0}$-value. However, the longitudinal residual stress shows a tension around the welded part, and the distribution profile is similar to the simulation. With respect to the transverse residual stress simulated by the FEM, the large tensile stresses exist in the area of the 1st and 2nd welding passes which are indicated in Fig 1 (b). The other tensile residual stresses exist in the right side of the U-notch 


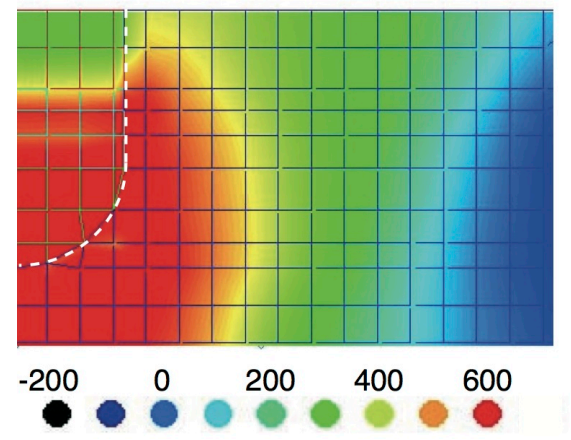

(a) $\sigma_{L}$

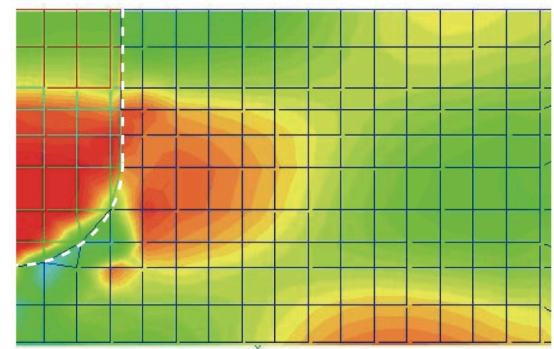

$-5050150250350$

(b) $\sigma_{T}$

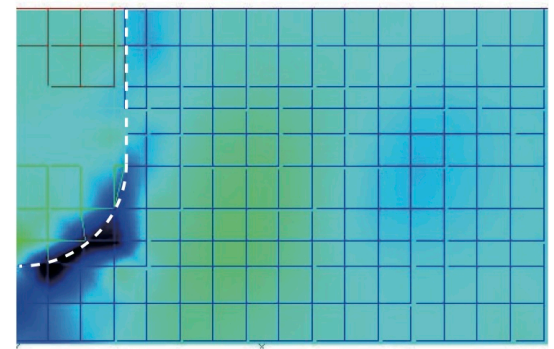

$-160-120-80-40 \quad 0 \quad 40 \quad 80$

(MPa)

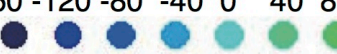

(c) $\sigma_{N}$

Fig. 8: Welding residual stresses simulated by finite elment method. (a) is longitudinal residual stress $\sigma_{L},(\mathrm{~b})$ is transverse residual stress $\sigma_{T}$ and (c) is normal residual stress. $\sigma_{L}, \sigma_{T}$ and $\sigma_{N}$ are defined in Fig. 7.

and near the back surface as shown in Fig. 8 (b). The distribution profile of the transverse residual stresses by the DSTM and the FEM are similar except the area of the 1st and 2nd welding passes.

The DSTM was applied to the stress measurement of the welded plate of austenitic stainless steel for the first time. It was useful to obtain the internal stress map of the welded austenitic stainless steel plate. Development of a 2-dimensional proportional detector for high energy is expected.

\section{Summary}

The welding specimen of austenitic stainless steel was prepared. The DSTM was applied to the stress measurement of the welding specimen using hard synchrotron X-ray of $70 \mathrm{keV}$. The obtained results are as follows:

(1) Although the detection efficiency of the PILATUS deteriorated due to high energy X-ray, the welding residual stress could be measured by DSTM over the exposure time for 30min.

(2) The diffraction profiles of the residual stresses corresponded with the FEM simulation, though the magnitude of the residual stresses by the DSTM was small as compared with that of the simulation.

The DSTM is useful to measure internal stress in welded materials. Development of a new detector for high energy X-rays is desired.

The authors acknowledge financial support by JSPS KAKENHI, Grant-in-aid for Challenging Exploratory Research (No. 24656083). The synchrotron radiation experiments were performed at the BL22XU in SPring-8 by the approval of Japan Atomic Energy Agency (No. 2012A-E02, No. 2012BE12, No. 2013A-E07).

\section{References}

[1] A.P. Reynolds, W. Tang, T. Gnaupel-Herold and H. Prask: Scripta Materialia, Vol. 48 (2003), pp. 1289-1294.

[2] A. Joseph, S.K. Rai, T. Jayakumar and N. Murugan: Inter. J. of Pressure Vessels and Piping, Vol. 82 (2005), pp. 700-705.

[3] K. Suzuki, T. Shobu and A. Shiro: Mater. Sci. Forum, Vols. 768-769 (2014) pp. 564-571.

[4] K. Suzuki, T. Shobu, A. Shiro and H. Toyokawa: Mater. Sci. Forum, Vol. 772 (2014), pp. 15-19.

[5] K. Suzuki, T. Shobu, A. Shiro and S. Zhang: Mater. Sci. Forum, Vol. 777 (2014), pp. 155-160.

[6] H.M. Ledbetter: Brit. J. of NDT, Vol. 23 (1981), pp. 286-287.

[7] K. Suzuki, A. Yamagishi, S. Nishikawa and T. Shobu: Maintenology, Vol. 11, No. 2 (2012), pp. 91-98. 